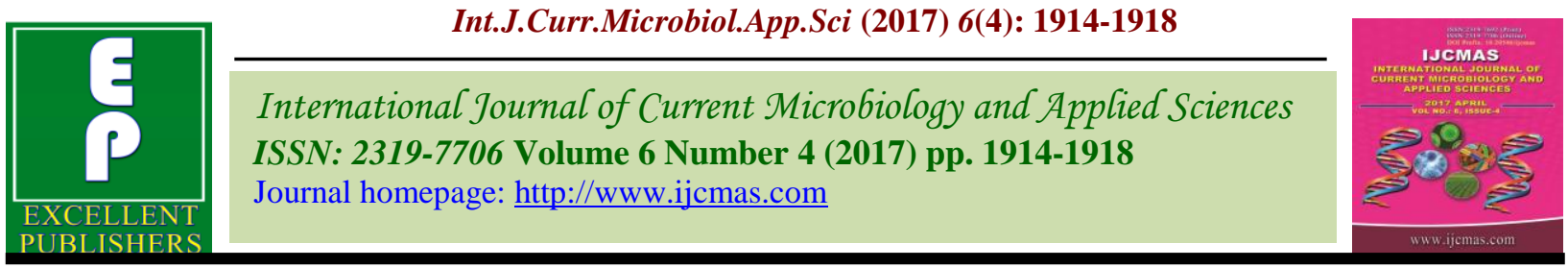

Original Research Article

https://doi.org/10.20546/ijcmas.2017.604.228

\title{
Stability Analysis for Quantitative Traits in Sugarcane (Saccharum officinarum)
}

\author{
R.B. Dubey, Baudh Bharti*, S.G. Khandagale and Khushbu Chittora \\ Department of Plant Breeding and Genetics, Maharana Pratap University of \\ Agriculture and Technology, Udaipur (Rajasthan)-313001, India \\ *Corresponding author
}

\begin{abstract}
A B S T R A C T
The experimental material comprising of seven varieties viz., Co 05009, CoH 05262,

\begin{tabular}{|c|}
\hline Keywords \\
\hline $\begin{array}{l}\text { Stability, G x E } \\
\text { interactions, } \\
\text { Quantitative traits, } \\
\text { Sugarcane } \\
\text { (Saccharam } \\
\text { officinarum). }\end{array}$ \\
\hline Article Info \\
\hline $\begin{array}{l}\text { Accepted: } \\
\text { 15 March } 2017 \\
\text { Available Online: } \\
10 \text { April } 2017\end{array}$ \\
\hline
\end{tabular}

CoH 05265, CoLk 05201, CoPk 05191, CoJ64(s) and CoPant84211(s) were grown in randomized block design with three replications, in three environments at Agriculture Research Station Ummedganj, Kota North west zone, India during 2009 to 2011. Mean squares due to genotypes were highly significant for tillers population (ha), NMC (ooo ha), cane length $(\mathrm{m})$, cane diameter $(\mathrm{cm})$, single cane weight $(\mathrm{kg})$, yield $(\mathrm{t} / \mathrm{ha})$, brix at 300 days, $\operatorname{ccs}(\mathrm{t} / \mathrm{ha})$ and brix at 250 days. However, Extraction \% and Sucrose \% at 240 days were no significant. Variety CoPk 05191 was found stable for NMC ('000 h), yield (t/ha) traits, except Tillers Population(ha), Cane Length (M), Cane Diameter (cm), Single Cane Weight (kg), Extraction (\%), BRIX\% at 240 Days, BRIX\% at 300 Days, Sucrose \% at 240 Days and CCS (t/ha), variety $\mathrm{CoH} 05265$ was found stable for Cane Diameter $(\mathrm{cm})$ and Single Cane Weight (kg) traits, except Tillers Population(ha), NMC ('000 h), Cane Length (M), Extraction (\%), yield(t/ha), BRIX\% at 240 Days, BRIX\% at 300 Days, Sucrose $\%$ at 240 Days and CCS (t/ha) while, variety $\mathrm{CoH} 05262$, was also found stable for Cane Diameter (cm) except remaining traits. Hence, these CoPk $05191 \mathrm{CoH} 05265$ and $\mathrm{CoH}$ 05262, promising lines could be recommended for commercial cultivation or could be suitability used in further improvement programme.
\end{abstract}

\section{Introduction}

The modern Saccharum spp. (cultivated sugarcane) is originated from complex hybridization events between Saccharum officinarum, S. Spontaneum, $S$ barberi, $S$ sinense and the related species (Sreenivasan et al., 1987). India is the second last producer of sugar after Brazil. It is propagated vegetatively for commercial planting by stem cuttings called 'sets'. Seed production of an elite sugarcane cultivar in sufficient quantity for planting in a vast area generally takes 10 15 years if multiplied through conventional methods of seed multiplication. Sugarcane is grown extensively in India. The crop occupies over 50.55 lakh hectares in the country with a production of 3481.87 lakh tonnes, of which $66 \%$ is concentrated in the northern states. Sugarcane in India is processed in to sugar, jaggery and khandsari. It forms an important item of Indian diet as a sweetening agent. White sugar contains mainly sucrose $(99.70 \%)$, where as jaggery has less sucrose $(51.00 \%)$ but it contains protein $(0.25 \%)$, glucose $(21.20 \%)$ and minerals $(3.40 \%)$ in 
addition to traces of fats (0.02 to $0.03 \%)$, calcium $(0.39 \%)$, vitamin $A$, vitamin $B$, phosphate. Sugarcane is an important and assured crop in tropical and subtropical India and the environment is always eratic in these tropics. This is need to breed genotypes for wide range of environments. Hence, knowledge of genotype $\mathrm{x}$ environment interactions is essential for such breeding programme, because potentiality of a genotype and stability of its performance can be judged by multi environmental test. Therefore, present experiment was undertaken to assess the stability of performance of varieties of sugarcane under three different environments (years).

\section{Materials and Methods}

The experimental material comprising of seven varieties viz., Co 05009, $\mathrm{CoH} 05262$, CoH 05265, CoLk 05201, CoPk 05191, CoJ64(s) and CoPant84211(s) were grown in randomized block design with replicated thrice at Research Station Ummedganj, Kota North west zone, India during 2009 to 2011. Each treatment plot comprised 4 rows of $6 \mathrm{~m}$ length spaced at $75 \mathrm{~cm}$ apart. The recommended agronomic packages of practices were followed to raise good fresh as well as ratoon crops. The observations were recorded for tillers population (ha), NMC ('000 h), Cane Length (M), Cane Diameter $(\mathrm{cm})$, Single Cane Weight $(\mathrm{kg})$, Extraction $(\%)$, yield(t/ha), BRIX\% At 240 Days, BRIX\% At 300 Days, Sucrose \% At 240 Days and CCS (t/ha). Phenotypic stability was estimated as per method given by Eberhart and Russells (1966).

\section{Results and Discussion}

The pooled analysis of variance for phenotypic stability (Table 1) revealed that the mean squares due to genotypes were highly significant for tillers population (ha),
NMC (ooo ha), cane length (m), cane diameter $(\mathrm{cm})$, single cane weight $(\mathrm{kg})$, yield (t/ha), brix at 300 days, ccs (t/ha) and brix at 250 days. However, Extraction \% and Sucrose $\%$ at 240 days were non significant indicating the presence of variability in the genotypes for the traits under study. The genotype $\mathrm{x}$ environment interaction was significant for cane length $(\mathrm{m})$, yield $(\mathrm{t} / \mathrm{ha})$ and $\operatorname{ccs}(\mathrm{t} / \mathrm{ha})$, while remaining traits were non significant. Queme et al., (2005) also reported that variance due to environment, genotype and $\mathrm{GxE}$ interactions were highly significant for cane yield, sucrose (\%) and sugar yield.

The environment linear components of variance were highly significant for all the traits, except the Sucrose \% at 240 days was non significant indicating that macro environmental differences were present under all the three environments. The mean squares due to linear component i.e. genotype $\mathrm{x}$ environment linear were significant for tillers population (ha), Cane length (M), cane diameter $(\mathrm{cm})$, single cane weight $(\mathrm{kg})$, yield (t/ha) and highly significant for ccs (t/ha) the traits. Mean squares due to pooled deviations (non-linear) were non significant for tillers population (ha), NMC (oooha), cane length $(\mathrm{M})$, cane diameter $(\mathrm{cm})$, single cane weight $(\mathrm{kg})$, yield (t/ha), brix at 240 days, brix at 300 days, ccs (t/ha) and Extraction \% traits. However, it was highly significant for Sucrose $\%$ at 240 days.

This suggested that both linear and non-linear components played important role in building up of total genotype $\mathrm{x}$ environment interactions for these traits. The significant mean squares due to pooled deviations for Sucrose $\%$ at 240 days trait indicated that genotypes differed considerably with respect to their stability and the prediction for this trait would be difficult. Kumar et al., (2007) reported significant GxE (linear) for cane yield and sugar yield. 
Int.J.Curr.Microbiol.App.Sci (2017) 6(4): 1914-1918

Table.1 Pooled analysis of variance for genotype x environment interactions for different traits in sugarcane

\begin{tabular}{|c|c|c|c|c|c|c|c|c|c|c|c|c|}
\hline \multirow[t]{3}{*}{ Source of variation } & \multirow[t]{3}{*}{ d.f } & \multicolumn{11}{|c|}{ Mean Sum of Squares } \\
\hline & & Tillers Population & NMC & Cane & Cane & Single Cane & Extractio & Yield (t/ha) & BRIX\% At & BRIX\% At & Sucrose \%At & CCS (t/ha) \\
\hline & & (000 ha) & ('000 ha) & Length (M) & Diameter $(\mathrm{cm})$ & Weight $(\mathrm{kg})$ & $\mathrm{n} \quad(\%)$ & & 240 Days & 300 Days & 240Days & \\
\hline Rep within Env. & 6 & 21.32 & 16.02 & 41.43 & 0.001 & $0.003^{*}$ & 0.37 & 13.63 & 0.05 & 0.02 & 0.03 & $0.21 *$ \\
\hline Varieties & 6 & $103.86^{* *}$ & $131.56 * *$ & $1093.40 * *$ & $0.04 * *$ & $0.03 * *$ & 0.81 & $262.05 * *$ & $0.16^{*}$ & $0.28 * *$ & 0.26 & $4.16^{* *}$ \\
\hline Env.+ (Var.x Env.) & 14 & $814.25 * *$ & $305.55^{* *}$ & $2210.09 * *$ & $0.12 * *$ & $0.05 * *$ & 1.03 & $513.39 * *$ & $0.14 *$ & 0.08 & 0.16 & $8.07 * *$ \\
\hline Environments & 2 & $5580.87 * *$ & $2070.54 * *$ & $13820.45^{* *}$ & $0.84 * *$ & $0.34 * *$ & $3.15^{*}$ & $3496.35 * *$ & $0.71 * *$ & $0.28 * *$ & 0.37 & $55.10 * *$ \\
\hline Var.x Env. & 12 & 19.81 & 11.38 & $275.03 *$ & 0.005 & 0.002 & 0.68 & $16.23^{*}$ & 0.04 & 0.04 & 0.12 & $0.23 *$ \\
\hline Environments (Lin.) & 1 & $11161.73 * *$ & $4141.07 * *$ & $27640.89^{* *}$ & $1.67 * *$ & $0.68 * *$ & $6.31 * *$ & $6992.70 * *$ & $1.43 * *$ & $0.56 * *$ & 0.74 & $110.21 * *$ \\
\hline Var.x Env.(Lin.) & 6 & $31.55^{*}$ & 5.55 & $464.09 *$ & $0.007 *$ & $0.003 *$ & 0.79 & $27.27^{*}$ & 0.04 & 0.05 & 0.07 & $0.40 * *$ \\
\hline Pooled Deviation & 7 & 6.92 & 14.76 & 73.69 & 0.002 & 0.001 & 0.48 & 4.45 & 0.03 & 0.03 & $0.15 * *$ & 0.04 \\
\hline Pooled Error & 36 & 38.87 & 22.15 & 71.05 & 0.003 & 0.003 & 0.39 & 13.76 & 0.03 & 0.04 & 0.02 & 0.18 \\
\hline Total & 20 & 601.13 & 253.35 & 1875.09 & 0.09 & 0.04 & 0.97 & 437.99 & 0.14 & 0.14 & 0.19 & 6.89 \\
\hline
\end{tabular}

$*$, ** Significance at $5 \%$ and $1 \%$ levels of probability respectively

Table.2 Stability Parameters of 11 traits in sugarcane (Eberhart and Russell's model 1966)

\begin{tabular}{|c|c|c|c|c|c|c|c|c|c|c|c|c|c|c|c|}
\hline \multirow[b]{2}{*}{ Genotypes } & \multicolumn{3}{|c|}{ Tillers Population (000 ha) } & \multicolumn{3}{|c|}{ NMC ('000 ha) } & \multicolumn{3}{|c|}{ Cane Length (M) } & \multicolumn{3}{|c|}{ Cane Diameter (cm) } & \multicolumn{3}{|c|}{ Single Cane Weight (kg) } \\
\hline & Mean & bi & $\mathbf{s}^{2} \mathbf{d i}$ & Mean & bi & $\mathbf{s}^{2} \mathbf{d i}$ & Mean & bi & $\mathbf{s}^{2} \mathbf{d i}$ & Mean & bi & $\mathbf{s}^{2} \mathbf{d i}$ & Mean & bi & $s^{2} \mathbf{d i}$ \\
\hline Co 05009 & 126.27 & 1.00 & -23.04 & 97.59 & 1.06 & -5.37 & 192.75 & 1.25 & -50.73 & 2.21 & 0.79 & 0.000 & 0.86 & 1.12 & -0.003 \\
\hline $\mathrm{CoH} 05262$ & 128.17 & 0.92 & -36.16 & 87.20 & 0.96 & -5.89 & 168.10 & 0.95 & 17.38 & 2.29 & 0.93 & 0.003 & 0.84 & 1.24 & -0.001 \\
\hline $\mathrm{CoH} 05265$ & 136.13 & 1.10 & -34.64 & 88.33 & 1.18 & -16.49 & 171.88 & 1.02 & -37.36 & 2.32 & 0.94 & -0.002 & 0.87 & 0.97 & -0.003 \\
\hline CoLk 05201 & 128.20 & $0.73 *$ & -36.15 & 86.95 & 0.89 & -19.66 & 171.09 & 1.12 & -5.79 & 2.29 & 0.80 & -0.002 & 0.80 & 0.99 & -0.003 \\
\hline Co Pk 05191 & 142.77 & 1.15 & -3.38 & 101.07 & $0.92 *$ & -21.26 & 199.18 & 1.44 & 152.02 & 2.26 & 1.23 & -0.001 & 0.98 & 1.04 & -0.003 \\
\hline Co J $64(\mathrm{~S})$ & 134.00 & $1.04 * *$ & -36.36 & 84.85 & 0.98 & -0.17 & 145.05 & 0.37 & 35.88 & 2.06 & 1.13 & -0.002 & 0.68 & 0.86 & -0.003 \\
\hline Co Pant $84211(\mathrm{~S})$ & 129.05 & 1.03 & -36.34 & 83.97 & 0.98 & 23.24 & 155.42 & 0.81 & -63.29 & 2.03 & 1.14 & 0.000 & 0.69 & $0.75^{*}$ & -0.003 \\
\hline Population Mean & 132.10 & & & 89.99 & & & 171.90 & & & 2.21 & & & 0.82 & & \\
\hline SE (mean) & 1.9 & & & 2.72 & & & 6.10 & & & 0.03 & & & 0.02 & & \\
\hline SE of bi & & 0.1 & & & 0.16 & & & 0.1 & & & 0.09 & & & 0.08 & \\
\hline
\end{tabular}

Table 2. Conted....

\begin{tabular}{|c|c|c|c|c|c|c|c|c|c|c|c|c|c|c|c|c|c|c|}
\hline \multirow[b]{2}{*}{ Genotypes } & \multicolumn{3}{|c|}{ Extraction (\%) } & \multicolumn{3}{|c|}{ Yield (t/ha) } & \multicolumn{3}{|c|}{ BRIX\% At 240 Days } & \multicolumn{3}{|c|}{ BRIX\% At 300 Days } & \multicolumn{3}{|c|}{ Sucrose \% At 240 Days } & \multicolumn{3}{|c|}{$\operatorname{CCS}(t / h a)$} \\
\hline & Mean & bi & $\mathrm{s}^{2} \mathrm{di}$ & Mean & bi & $\mathrm{s}^{2} \mathrm{di}$ & Mean & bi & $\mathbf{s}^{2} \mathrm{di}$ & Mean & bi & $\mathbf{s}^{2} \mathbf{d i}$ & Mean & bi & $\mathbf{s}^{2} \mathbf{d i}$ & Mean & bi & $\mathbf{s}^{2} \mathbf{d i}$ \\
\hline Co 05009 & 51.81 & 1.10 & -0.15 & 74.13 & $1.09 *$ & -13.72 & 17.72 & 1.73 & -0.003 & 19.99 & 1.58 & -0.04 & 15.31 & 1.40 & 0.03 & 8.58 & $1.11 *$ & -0.18 \\
\hline $\mathrm{CoH} 05262$ & 52.01 & 0.21 & 0.57 & 71.03 & $1.24 * *$ & -13.74 & 18.18 & 0.30 & -0.02 & 20.02 & 0.44 & -0.03 & 15.29 & -0.20 & $0.49 * *$ & 8.47 & $1.23 *$ & -0.18 \\
\hline $\mathrm{CoH} 05265$ & 52.15 & 0.89 & -0.26 & 75.01 & 1.09 & -6.40 & 17.83 & 0.62 & 0.01 & 20.04 & 1.91 & -0.04 & 15.07 & 1.04 & -0.02 & 8.83 & 1.11 & -0.07 \\
\hline CoLk 05201 & 52.42 & 0.42 & -0.34 & 68.92 & 0.97 & -12.70 & 18.37 & 1.07 & 0.01 & 19.88 & 1.27 & -0.04 & 15.37 & 0.86 & -0.02 & 8.14 & 0.99 & -0.13 \\
\hline Co Pk 05191 & 51.72 & 0.05 & -0.31 & 82.94 & 0.97 & -4.43 & 18.23 & 1.05 & -0.02 & 20.53 & -0.46 & 0.09 & 16.02 & 0.20 & $0.11 *$ & 9.79 & 0.91 & -0.12 \\
\hline Co J 64 (S) & 52.86 & 2.77 & 0.02 & 55.06 & 0.73 & -2.40 & 18.00 & 1.31 & -0.01 & 19.59 & 1.42 & 0.01 & 15.49 & 1.77 & $0.21 * *$ & 6.31 & 0.77 & -0.11 \\
\hline Co Pant $84211(\mathrm{~S})$ & 53.09 & 1.53 & 1.17 & 60.52 & 0.87 & -11.60 & 17.92 & 0.88 & 0.04 & 19.68 & 0.82 & -0.02 & 15.43 & 1.90 & $0.12 * *$ & 6.92 & 0.86 & -0.18 \\
\hline Population Mean & 52.30 & & & 69.67 & & & 18.04 & & & 19.97 & & & 15.43 & & & 8.15 & & \\
\hline SE (mean) & 0.49 & & & 1.49 & & & 0.12 & & & 0.12 & & & 0.27 & & & 0.15 & & \\
\hline $\mathrm{SE}$ of bi & & 0.73 & & & 0.07 & & & 0.39 & & & 0.60 & & & 1.19 & & & 0.05 & \\
\hline
\end{tabular}

*, ** Significance at $5 \%$ and $1 \%$ levels of probability respectively 
In the present investigation, both predictable (linear) and unpredictable (deviation) component were significant and shared the genotype $\mathrm{x}$ environments interaction. However, predictable component was predominant for some of the traits, while unpredictable component was predominant for some other traits.

A perusal of phenotypic stability parameters (Table 2) for Tillers Population / ha, NMC ('000 h), Cane Length (m), Cane Diameter $(\mathrm{cm})$, Single Cane Weight $(\mathrm{kg})$, yield (t/ha), Extraction (\%), BRIX\% at 240 Days, BRIX\% at 300 Days, Sucrose \% at 240 Days and CCS (t/ha) (Table 2.) revealed that only three varieties namely $\mathrm{CoPk} 05191 \mathrm{CoH} 05262$, and $\mathrm{CoH} 05265$ were exhibited $\mathrm{S}^{2} \mathrm{di}$ (deviation from regression) non-significant and regression coefficient (bi) equal to unity (bi $\approx 1)$ with mean value higher than the population mean. Similar results have been reported earlier Tahir et al.(2013) an Guddadamath et al., (2014) for cane yield and Tiawari et al., (2011) for NMC. These varieties were therefore, considered suitable and stable under variable environmental conditions, variety CoPk 05191 for NMC ('000 h), yield (t/ha), variety $\mathrm{CoH} 05262$, for Cane Diameter $(\mathrm{cm})$ and variety $\mathrm{CoH} 05265$ for Cane Diameter $(\mathrm{cm})$ and Single Cane Weight (kg) traits. Variety $\mathrm{CoH} 05262$ was also found suitable and stable under unfavorable environment for BRIX\% at 240 Days and BRIX\% at 300 Days. All seven varieties were exhibited $S^{2}$ di (deviation from regression) non-significant, regression coefficient (bi) greater than unity (bi>1) with mean value higher than the population mean. All these varieties were considered suitable and stable under favourable environmental conditions for NMC ('000 h), Cane Length (m), Single Cane Weight $(\mathrm{kg})$, yield (t/ha), CCS (t/ha, Co 05009), Single Cane Weight (kg), yield (t/ha), and CCS (t/ha, CoH 05262), tillers population(ha), yield (t/ha) BRIX\% at
300 Days and CCS (t/ha $\mathrm{CoH}$ 05265), BRIX\% at 240 Days (CoLk 05201), tillers population(ha), Cane Length (M), Cane Diameter $(\mathrm{cm})$ Single Cane Weight $(\mathrm{kg})$, BRIX\% at 240 Days (CoPk 05191), Extraction (\%) and Sucrose \% at 240 Days (CoJ64(s)and CoPant84211(s), while the three varieties namely, (CoLk 05201), $\mathrm{CoH} 05262$ and (CoPk 05191), were exhibited $\mathrm{S}^{2}$ di nonsignificant, regression coefficient less than unity (bi<1) with mean value higher than the population mean, thereby indicating its stability and suitability under unfavorable environmental conditions for Cane Diameter (cm) and Extraction (\%) CoLk 05201). BRIX\% at 300 Days (CoH 05262) and Sucrose \% at 240 Days (CoPk 05191). Variety CoPk 05191 was found stable for NMC ('000 h), yield (t/ha) traits, except Tillers Population (ha), Cane Length (M), Cane Diameter $(\mathrm{cm})$, Single Cane Weight (kg), Extraction (\%), BRIX\% at 240 Days, BRIX\% at 300 Days, Sucrose \% at 240 Days and CCS (t/ha), variety $\mathrm{CoH} 05265$ was found stable for Cane Diameter $(\mathrm{cm})$ and Single Cane Weight (kg) traits, except Tillers Population (ha), NMC ('000 h), Cane Length (M), Extraction (\%), yield(t/ha), BRIX\% at 240 Days, BRIX\% at 300 Days, Sucrose \% at 240 Days and CCS (t/ha) while, variety $\mathrm{CoH}$ 05262, was also found stable for Cane Diameter $(\mathrm{cm})$ except remaining traits. Therefore, these promising $\mathrm{CoPk} 05191 \mathrm{CoH}$ 05265 and $\mathrm{CoH}$ 05262, lines could be recommended for commercial cultivation or could be suitability used in further improvement programme.

\section{References}

Eberhart, S.A., Russel, W.A. 1966. Stability parameters for comparing varieties. Crop Sci., 6: 36-40.

Guddadamath, S.S., Patil, S.B., Khadi, B.M. 2014. Stability analysis of cane and jaggery yield in elite sugarcane 
genotypes (Saccharum spp.). Indian $J$. Genet., 74(2): 261-264.

Kumar, S., Singh, J., Singh, P.K., Pandey, D.K. 2007. Stability of yield and its component characters in sugarcane (Saccharum spp hybrid complex). Indian J. Agri. Sci., 77(4): 220-223.

Queme, J.L., Orozco, H., Ovalle, W., Melgar, M. 2005. Analysis of genotype by environment interaction for sugarcane based on the AMMI model. Sugarcane Int., 23(4): 21-24.

Sreenivasan, T.V., Ahloowalia, B.S., Heinz, D.J. 1987. Cytogenetics. In Sugarcane Improvement Through Breeding(Heinz
D.J., ed.), Elsevier, Amsterdam, pp. 211-253.

Tahir, M., Rahman, H., Amjad, A., Anwar, S., Khalid, M. 2013. Assessment of genotype $\times$ environment interaction and stability of promising sugarcane genotypes for different agronomic characters in Peshawar valley. Am. $J$. Exp. Agric., 3 (1): 142-151.

Tiawari, D.K., Pandey, P., Singh, R.K., Singh, S.P., Singh, S.B. 2011. Genotype $\mathrm{x}$ environment interaction and stability analysis of elite clones of sugarcane. Int. J. Pl. Br. and Gen., 5(1): 93-98.

\section{How to cite this article:}

Dubey, R.B., Baudh Bharti, S.G. Khandagale and Khushbu Chittora. 2017. Stability Analysis for Quantitative Traits in Sugarcane (Saccharum officinarum). Int.J.Curr.Microbiol.App.Sci. 6(4): 1914-1918. doi: https://doi.org/10.20546/ijcmas.2017.604.228 